\title{
Capturing the National Landscape of University Departments Housing MLS Programs and Analysis of Student Outcomes across Three Novel Models
}

\author{
Virginia C. Hughes* \\ Medical and Molecular Sciences, University of Delaware, Newark, USA \\ *Corresponding author: vhughes@udel.edu
}

Received December 02, 2019; Revised January 05, 2020; Accepted January 15, 2020

\begin{abstract}
University-based MLS programs accredited by NAACLS were categorized into three novel department models (Singular, Horizontal, and Vertical). Average 3-yr ASCP BOC pass rates and graduation rates were accessed from the MLS program website. Analysis of variance statistics was performed on BOC pass rates and graduation rates across the three department models. In addition, a Pearson correlation coefficient was derived assessing the association between the number of programs offered in Vertical departments and BOC pass rates and graduation rates. There was not a statistically significance difference found among department models with regard to BOC pass rates or graduation rates. The Pearson correlation coefficient demonstrated a positive correlation $(\mathrm{r}=.04, \mathrm{P}>.05)$ ) when assessing the association between number of programs in the Vertical model and average three-year graduation rates. However, the Pearson correlation coefficient yielded a negative correlation with respect to the number of programs offered in the Vertical model and average three-year BOC pass rates $(r=-.26, P<.05)$. This statistically significant negative correlation indicates lower BOC pass rates with increasing numbers of programs. As the trend toward Vertical departments increase college administrators will need to continually assess resources allocated to programs in departments while employing strategies that yield favorable student outcomes.
\end{abstract}

Keywords: analysis of variance, correlation coefficient, student outcomes

Cite This Article: Virginia C. Hughes, "Capturing the National Landscape of University Departments Housing MLS Programs and Analysis of Student Outcomes across Three Novel Models." American Journal of Educational Research, vol. 8, no. 1 (2020): 35-45. doi: 10.12691/education-8-1-6.

\section{Introduction}

Since the first baccalaureate program in Medical Technology (MT) was started at the University of Minnesota in 1922, Medical Laboratory Science programs (MLS) as they as now called have strived for producing qualified graduates who are skilled in all areas of the clinical laboratory and provide clinicians with laboratory data which aid in diagnosing and treating patients. MLS programs may be housed in hospitals or universities/colleges. In the university setting, MLS programs may be organized into departments or schools as part of a compartmentalized college structure. The academic department can be described as the focal point of academic expertise in a given discipline [1]. Ideally, it provides an environment and resources that cultivates scholarly pursuits of both faculty and students guided by a mission and goals for continued growth that align with that of the college and university. Universities are not unlike other organizations regarding change. Change in higher education with subsequent alterations to departments may be influenced by internal and external constituencies, and tends to be more successful when aligned with the institution's culture [2].

Internal constituencies comprise faculty, students, staff, administrators, and governing boards. External constituencies include parents, the public, elected government leaders, the press, business owners, and foundations. Factors related to MLS programs that can elicit change include new leadership, decreased student enrollment, low BOC pass rates and graduation rates, loss of clinical affiliates and/or accreditation, faculty departures, and decreased funding. Medical Laboratory Science programs are one of the most expensive programs to operate in the university setting; in an economic downturn they are one of the first programs targeted for closure. According to the National Accrediting Agency for Clinical Laboratory Sciences (NAACLS) the number of MLS programs in the United States have decreased by 25\% since 1990 . [3] To circumvent the risk of program closure due to scant enrollment and/or high cost associated with MLS programs university administrators may choose to relocate an MLS program into another department or college or add additional degree programs to the current department thought to be associated with high enrollment. 
The purpose of this study is to categorize MLS programs housed in universities across the United States into three novel academic department models and assess how these models affect student outcomes such ASCP BOC pass rates and graduation rates. These two parameters are of cardinal importance to the success and growth of MLS programs reflecting both the quality of teaching and adequacy of resources. The departmental model employed may necessitate sharing of resources across disciplines and diversion of teaching responsibilities which may impact student outcomes. Charting the current landscape of MLS programs in academic departments may provide insight to department chairs and program directors into sustainability of stand-alone and interdisciplinary models and ideas for the future.

\section{Study Design}

\subsection{Identification of MLS Programs}

Current NAACLS accredited university-based MLS programs were identified on the NAACLS website (www.naacls.org) using filters of state and type of program (medical laboratory scientist). A link was provided to the MLS programs' website where the name of department, college, programs in the department, 3-yr BOC pass rates and graduation rates were accessed. Hospital-based MLS programs and those not housed in an academic department were excluded from this study.

\subsection{Definition and Assignment of Department Models: Singular, Horizontal, Vertical}

The Singular model is defined as a department which only offers a baccalaureate degree in MLS; there are no minor degrees, graduate degrees, or certificate programs (except post-baccalaureate MLS certificate) offered. A department was also coded as Singular if it offered an on campus MLS program and online MLS program. The Horizontal model is defined as a department that offers a baccalaureate degree in MLS and at least one other baccalaureate degree program. The Vertical model is defined as a department that offers a baccalaureate degree in MLS and at least one other non-baccalaureate program such as a certificate program, minor degree, Master's degree, and/or doctorate degree.

\subsection{Statistics}

A Pearson Correlation Coefficient (r) was determined using the number of programs in Vertical departments (independent variable) and 3-yr BOC pass rates (dependent variable). In addition, $r$ was also determined using the number of programs in Vertical departments (independent variable) and 3-yr Graduation rate (dependent variable). A one-way ANOVA was conducted to determine if there was a statistical difference between the three models with regard to the 3-yr graduation rate and 3-yr ASCP BOC pass rate. The statistical program employed was SPSS.

\section{Results}

\subsection{Department Models}

A total of 86 MLS NAACLS accredited programs are housed in an academic department; 14 were categorized as Singular, 6 as Horizontal, and 66 as Vertical (Table 1 and Table 2). Figure 1 depicts departments where MLS programs are housed among the three models, and Figure 2 depicts colleges and their respective departments. As shown in Table 1, names of programs vary from MLS, CLS, or Medical Technology in the Singular group. Departments in the Horizontal group offer a variety of baccalaureate programs ranging from life science programs like biochemistry and biology to allied health programs like radiological sciences at McNeese State University and Histotechnology at University of Arkansas. Table 2 demonstrates the heterogenous landscape of the Vertical department model. Other than all departments offering a baccalaureate degree in MLS, there is no identifiable pattern of certificate programs, minors, bachelors (other than MLS), masters, or doctorate degrees across the United States. Figure 1 depicts names of departments among the three models housing MLS programs with the frequency of each department in parentheses. As expected, department names in the Singular group are named MLS, MT, or CLS. The Horizontal group has 6 departments with the Biology department being the most common. In the Vertical group the most common department name is either MLS or CLS, followed by Allied Health, Health Sciences and Biology, and Pathology. Figure 2 depicts Colleges and Schools with respective departments housing MLS programs. Just as there is great variation in department names the same can be said for Colleges and Schools. Most departments that house MLS programs are in the College of Health Sciences.

\subsection{ASCP BOC Pass Rate}

Figure 3 depicts the average ASCP BOC pass rates for the past three years among Singular, Horizontal, and Vertical models. A one-way ANOVA revealed there was not a significant difference among the three groups with regard to the ASCP BOC pass rates. The average $3-y r$ ASCP BOC pass rates for Singular $(n=12)$, Horizontal $(n=5)$, and Vertical $(n=61)$ were $89.4 \%$, $80.5 \%$, and $89.9 \%$, respectively. Figure 4 depicts a scatterplot and correlation coefficient of number of programs within vertical departments and their 3-yr BOC pass rates. There was a negative correlation, $\mathrm{r}=-.26$ between the number of programs offered in the vertical model with the average 3 yr ASCP BOC pass rate which was statistically significant, $P=.02$. This can be interpreted as the more programs a department offers the lower the BOC pass rate.

\subsection{Graduation Rate}

Figure 5 shows a scatterplot of correlation coefficient of the number of programs within vertical departments and their average 3-yr graduation rates. There was a positive correlation between number of programs in a department 
and graduation rate $(\mathrm{r}=.04)$; however, results were not statistically significant, $\mathrm{P}>.05$. A one-way ANOVA did not yield significance among the three types of models and 3-yr graduation rate $(\mathrm{P}>.05)$. The average 3-yr graduation rates for Singular $(n=12)$, Horizontal $(n=6)$, and Vertical (n=59) were 92.6, 99, and 95.7\%, respectively.

Table 1. Programs in Singular and Horizontal Departments

\begin{tabular}{|l|l|c|c|}
\hline Singular & BS Programs & \multicolumn{1}{|c|}{ University } & BS Programs \\
\hline University & MLS & McNeese State University & MLS, Radiologic Science \\
\hline Auburn University @Montgomery & MLS & Eastern Michigan University & $\begin{array}{c}\text { MLS, Cytogenetics, Histotech, Clinical Lab } \\
\text { Studies }\end{array}$ \\
\hline University of Arkansas Medical Science & CLS & East Carolina University & CLS, BIO \\
\hline Howard University & CLS & Mercy College & CLS, BIO, Health Science, Exercise Science \\
\hline University of West Florida & $\begin{array}{l}\text { Medical } \\
\text { Technology }\end{array}$ & $\begin{array}{c}\text { Oklahoma Christian } \\
\text { University }\end{array}$ & $\begin{array}{c}\text { MLS, Biochemistry, BIO, Cell/Mol BIO, Nutrition } \\
\text { and Exercise Science, Science Education }\end{array}$ \\
\hline University of Hawaii-Manoa & MLS & Neumann University & CLS \\
\hline Wichita State University & MLS & & \\
\hline Eastern Kentucky University & CLS & & \\
\hline LSU-Health Sciences & MLS & & \\
\hline University of MASS-Dartmouth & CLS & & \\
\hline Winston-Salem University & Medical & & \\
\hline Marist College & MLS & & \\
\hline Oregon Tech & CLS & & \\
\hline Marquette University & MT & & \\
\hline Pontifical Catholic University of Puerto Rico & & & \\
\hline
\end{tabular}

MLS (Medical Laboratory Science), CLS (Clinical Laboratory Science), MT (Medical Technology), BIO (Biology).

Table 2. Programs in Vertical Departments

\begin{tabular}{|c|c|c|c|c|c|c|}
\hline University & Certificate & Minor & AA & BS & MS & Doctoral \\
\hline $\begin{array}{c}\text { University of } \\
\text { Alaska } \\
\text { (Anchorage) }\end{array}$ & $\begin{array}{c}\text { PBT, } \\
\text { Clinical } \\
\text { Assisting } \\
\end{array}$ & & MLT & MLS & & \\
\hline $\begin{array}{l}\text { Tuskegee } \\
\text { University }\end{array}$ & & & & MLS & OT & \\
\hline $\begin{array}{l}\text { University of } \\
\text { Alabama } \\
\text { (Birmingham) }\end{array}$ & $\begin{array}{l}\text { Biotech } \\
\text { Regulatory } \\
\text { Affairs }\end{array}$ & $\begin{array}{l}\text { Biomedical } \\
\text { Sciences }\end{array}$ & & $\begin{array}{l}\text { CLS, Biomedical } \\
\text { Science }\end{array}$ & $\begin{array}{l}\text { CLS, Nuclear Medicine } \\
\text { Technology, Genetic } \\
\text { Counseling, Health } \\
\text { Physics, PA, Biomedical } \\
\text { and Health Science, } \\
\text { Biotechnology }\end{array}$ & Pharmacy \\
\hline $\begin{array}{c}\text { Arkansas State } \\
\text { University }\end{array}$ & & & CLS & CLS & & \\
\hline $\begin{array}{l}\text { Loma Linda } \\
\text { University }\end{array}$ & PBT & & & MLS, Cytotech & & \\
\hline University of CT & $\begin{array}{c}\text { Post-bac MLS, } \\
\text { DGS }\end{array}$ & & & $\begin{array}{l}\text { MLS, DGS, Allied } \\
\text { Health Sciences, } \\
\text { Dietetics }\end{array}$ & $\begin{array}{c}\text { Health Promotion } \\
\text { Science, Healthcare } \\
\text { Genetics, Genetic } \\
\text { Counseling } \\
\end{array}$ & $\begin{array}{l}\text { Health Promotion } \\
\text { Science }\end{array}$ \\
\hline $\begin{array}{l}\text { George Washington } \\
\text { University }\end{array}$ & Post-bac MLS & & & MLS & $\begin{array}{l}\text { Molecular Diagnostics, } \\
\text { Health Sciences (Lab } \\
\text { Medicine, } \\
\text { Immunohematology and } \\
\text { Biotechnology, Clinical } \\
\text { Microbiology), } \\
\text { Translational } \\
\text { Microbiology } \\
\end{array}$ & \\
\hline $\begin{array}{l}\text { University of } \\
\text { Delaware }\end{array}$ & $\begin{array}{c}\text { Molecular } \\
\text { Diagnostics, } \\
\text { Post-bac MLS }\end{array}$ & $\begin{array}{c}\text { Forensic } \\
\text { Science, Genetic } \\
\text { Counseling, } \\
\text { Medical } \\
\text { Diagnostics, } \\
\text { Molecular } \\
\text { Diagnostics }\end{array}$ & & $\begin{array}{c}\text { MLS, Medical } \\
\text { Diagnostics, } \\
\text { Medical Diagnostics } \\
\text { Pre-PA, Applied } \\
\text { Molecular Biology } \\
\text { and Biotechnology }\end{array}$ & $\begin{array}{l}\text { Medical Sciences, MLS, } \\
\text { Applied Molecular } \\
\text { Biology and } \\
\text { Biotechnology }\end{array}$ & Medical Sciences \\
\hline $\begin{array}{l}\text { Florida Gulf Coast } \\
\text { University }\end{array}$ & $\begin{array}{l}\text { Post-bac MLS, } \\
\text { Health Service } \\
\text { Admin }\end{array}$ & & & $\begin{array}{l}\text { CLS, Community } \\
\text { Health, Public } \\
\text { Health }\end{array}$ & Health Science, PA & \\
\hline $\begin{array}{l}\text { Georgia Southern } \\
\text { University }\end{array}$ & $\begin{array}{l}\text { Advanced } \\
\text { Imaging, } \\
\text { Nuclear Med, } \\
\text { Radiation } \\
\text { Therapy }\end{array}$ & & & $\begin{array}{c}\text { MLS, Radiologic } \\
\text { Science, Respiratory } \\
\text { Therapy }\end{array}$ & & \\
\hline
\end{tabular}




\begin{tabular}{|c|c|c|c|c|c|c|}
\hline University & Certificate & Minor & AA & BS & MS & Doctoral \\
\hline Rush University & SBB & & & & $\begin{array}{l}\text { MLS, Clinical Lab } \\
\text { Management }\end{array}$ & \\
\hline $\begin{array}{l}\text { Illinois State } \\
\text { University }\end{array}$ & $\begin{array}{l}\text { Industrial } \\
\text { Hygienist, } \\
\text { QEP, HMM }\end{array}$ & $\begin{array}{c}\text { Health } \\
\text { Education, } \\
\text { Public Health }\end{array}$ & & $\begin{array}{l}\text { MLS, Safety, Health } \\
\text { Promotion/ED, } \\
\text { HIM, } \\
\text { Environmental } \\
\text { Health }\end{array}$ & & \\
\hline $\begin{array}{l}\text { University of } \\
\text { Illinois Springfield }\end{array}$ & & & & $\begin{array}{c}\text { Clinical and } \\
\text { Medical Lab } \\
\text { Science, Exercise } \\
\text { Science }\end{array}$ & Athletic Training & \\
\hline Indiana University & & & Histotech & $\begin{array}{c}\text { CLS, } \\
\text { Cytotechnology } \\
\end{array}$ & Pathology Assistant & $\begin{array}{c}\text { Experimental } \\
\text { Pathology } \\
\end{array}$ \\
\hline $\begin{array}{l}\text { University of } \\
\text { Kansas }\end{array}$ & & & & CLS & $\begin{array}{l}\text { Molecular and. } \\
\text { Biotechnology }\end{array}$ & CLS \\
\hline $\begin{array}{c}\text { Bellaramine } \\
\text { University }\end{array}$ & & & & $\begin{array}{l}\text { MLS, Health } \\
\text { Science }\end{array}$ & Health Science & \\
\hline $\begin{array}{l}\text { University of } \\
\text { Kentucky }\end{array}$ & UGRHH & & & $\begin{array}{l}\text { MLS, MLT-MLS } \\
\text { online, Human } \\
\text { Health Science }\end{array}$ & & \\
\hline LSU Alexandria & Pharm Tech & & $\begin{array}{c}\text { MLS, } \\
\text { Radiologic } \\
\text { Technology }\end{array}$ & $\begin{array}{l}\text { MLS, Elder Care } \\
\text { Administration, } \\
\text { Health Professions } \\
\text { (concentration in } \\
\text { Cardiac Device } \\
\text { Sales Marketing, } \\
\text { Cardiovascular } \\
\text { Technology), Health } \\
\text { Science Medical } \\
\text { Imaging, General } \\
\text { Studies with } \\
\text { concentration in } \\
\text { Health Science }\end{array}$ & & \\
\hline $\begin{array}{l}\text { LSU Health } \\
\text { Sciences } \\
\text { Shreveport } \\
\end{array}$ & & & & $\begin{array}{c}\text { MLS, } \\
\text { Cardiopulmonary } \\
\text { Science } \\
\end{array}$ & PA & \\
\hline $\begin{array}{l}\text { University of } \\
\text { Massachusetts } \\
\text { Lowell }\end{array}$ & $\begin{array}{l}\text { Clinical Path, } \\
\text { Pharm Science }\end{array}$ & & & $\begin{array}{l}\text { MLS, Nutritional } \\
\text { Science, } \\
\text { Pharmaceutical } \\
\text { Science } \\
\end{array}$ & $\begin{array}{l}\text { MLS, Pharmaceutical } \\
\text { Science }\end{array}$ & $\begin{array}{l}\text { Pharmaceutical } \\
\text { Science }\end{array}$ \\
\hline $\begin{array}{c}\text { Morgan State } \\
\text { University }\end{array}$ & & & & $\begin{array}{c}\text { Medical } \\
\text { Technology, BIO }\end{array}$ & BIO, Science and ED & $\begin{array}{c}\text { Bioenvironmental } \\
\text { Science } \\
\end{array}$ \\
\hline $\begin{array}{l}\text { University of } \\
\text { Maryland } \\
\text { Baltimore }\end{array}$ & $\begin{array}{c}\text { Categorical } \\
\text { Blood Bank, } \\
\text { Hematology, } \\
\text { Chemistry, } \\
\text { MicroBIO }\end{array}$ & & & $\begin{array}{l}\text { Medical and } \\
\text { Research } \\
\text { Technology, } \\
\text { Biotechnology }\end{array}$ & $\begin{array}{l}\text { Biomedical Research, } \\
\text { Laboratory Management }\end{array}$ & \\
\hline $\begin{array}{c}\text { Andrews } \\
\text { University }\end{array}$ & Post-bac MLS & & & $\begin{array}{l}\text { MLS, Allied Health } \\
\text { Administration }\end{array}$ & MLS & \\
\hline $\begin{array}{l}\text { Michigan } \\
\text { Technology } \\
\text { University }\end{array}$ & $\begin{array}{l}\text { Secondary ED } \\
\text { Teaching }\end{array}$ & $\begin{array}{c}\text { Biochem, Mol } \\
\text { BIO, } \\
\text { BioprocessEng, } \\
\text { Ecology, } \\
\text { FishBIO, Micro, } \\
\text { Nanoscale } \\
\text { ScienceEng, } \\
\text { PlantBIO, Plant } \\
\text { Science, Remote } \\
\text { Sensing } \\
\end{array}$ & & $\begin{array}{c}\text { MLS, } \\
\text { Cytotechnology, } \\
\text { BIO, Biochemical } \\
\text { and Molecular BIO, } \\
\text { Bioinformatics }\end{array}$ & $\mathrm{BIO}$ & BIO, Biochemistry \\
\hline $\begin{array}{l}\text { Saginaw Valley } \\
\text { State University }\end{array}$ & Gerontology & $\begin{array}{c}\text { Emergency } \\
\text { Management, } \\
\text { Gerontology, } \\
\text { Health Science }\end{array}$ & & $\begin{array}{l}\text { MLS, Health } \\
\text { Science, } \\
\text { Neuroscience, } \\
\text { Public Health }\end{array}$ & & \\
\hline $\begin{array}{l}\text { Wayne State } \\
\text { University }\end{array}$ & $\begin{array}{c}\text { Forensic } \\
\text { Investigation }\end{array}$ & & & $\begin{array}{l}\text { CLS, Mortuary } \\
\text { Science }\end{array}$ & Pathologist Assistant & \\
\hline $\begin{array}{l}\text { St. Louis } \\
\text { University }\end{array}$ & & & & $\begin{array}{l}\text { MLS (pre-Med, Pre- } \\
\text { PA), Health } \\
\text { Information } \\
\text { Management, } \\
\text { Health Science, } \\
\text { Investigational and } \\
\text { Medical Science, } \\
\text { MRI, Nuclear } \\
\text { Medicine } \\
\text { Technology }\end{array}$ & $\begin{array}{l}\text { Molecular Imaging and } \\
\text { Therapeutics }\end{array}$ & \\
\hline
\end{tabular}




\begin{tabular}{|c|c|c|c|c|c|c|}
\hline University & Certificate & Minor & $\mathbf{A A}$ & BS & MS & Doctoral \\
\hline $\begin{array}{l}\text { University of } \\
\text { Southern } \\
\text { Mississippi } \\
\end{array}$ & & & & $\begin{array}{l}\text { MLS, MLT-MLS } \\
\text { online }\end{array}$ & MLS & \\
\hline $\begin{array}{l}\text { Montana State } \\
\text { University }\end{array}$ & & $\begin{array}{c}\text { Non-Teaching } \\
\text { Genetics/Micro } \\
\text { BIO }\end{array}$ & & $\begin{array}{c}\text { MLS, Biotech } \\
\text { Animal Science, } \\
\text { Biotech Microbial } \\
\text { Systems, Env } \\
\text { Health, Micro, Env } \\
\text { Micro, Pre-Med, } \\
\text { Pre-Vet } \\
\end{array}$ & & \\
\hline $\begin{array}{c}\text { University of NC } \\
\text { Chapel Hill }\end{array}$ & & & & CLS & CLS & \\
\hline $\begin{array}{l}\text { University of New } \\
\text { Hampshire }\end{array}$ & & $\begin{array}{c}\text { Biochemistry, } \\
\text { Mol/Cell BIO, } \\
\text { Biomed Science, } \\
\text { Genetics }\end{array}$ & & $\begin{array}{l}\text { MLS, Biochemistry, } \\
\text { Molecular and Cell } \\
\text { BIO, Biomedical } \\
\text { Science, Medical } \\
\text { and Veterinary } \\
\text { Science, Medical } \\
\text { MicroBIO }\end{array}$ & $\begin{array}{l}\text { Biochemistry, Genetics, } \\
\text { Microbiology }\end{array}$ & $\begin{array}{l}\text { Biochemistry, } \\
\text { Genetics, } \\
\text { MicroBIO, } \\
\text { Molecular and } \\
\text { Evolutionary } \\
\text { Systems }\end{array}$ \\
\hline Rutgers University & $\begin{array}{c}\text { Post-bac MLS, } \\
\text { Cardiac } \\
\text { Sonography, } \\
\text { PBT, } \\
\text { Diagnostic } \\
\text { Medical } \\
\text { Sonography, } \\
\text { Nuclear Med } \\
\text { Technology, } \\
\text { Radiologic } \\
\text { Imaging } \\
\text { Modalities } \\
\end{array}$ & & & $\begin{array}{c}\text { CLS, Cardiac } \\
\text { Sonography, } \\
\text { Diagnostic Medical } \\
\text { Sonography, } \\
\text { Nuclear Medicine } \\
\text { Technology, } \\
\text { Medical Imaging } \\
\text { Science }\end{array}$ & $\begin{array}{l}\text { CLS, Cytopathology, } \\
\text { Radiologic Assistant }\end{array}$ & CLS \\
\hline $\begin{array}{l}\text { University of New } \\
\text { Mexico }\end{array}$ & & & & MLS & MLS & \\
\hline $\begin{array}{c}\text { College of } \\
\text { Southern Nevada }\end{array}$ & $\begin{array}{c}\text { Dental } \\
\text { Assisting }\end{array}$ & & $\begin{array}{c}\text { MLT, } \\
\text { Radiologic } \\
\text { Therapy, } \\
\text { PT } \\
\text { Assistant, } \\
\text { Ophthalmic } \\
\text { Tech, } \\
\text { Dental } \\
\text { Hygiene } \\
\end{array}$ & $\begin{array}{l}\text { MLS, Dental } \\
\text { Hygiene }\end{array}$ & & \\
\hline $\begin{array}{l}\text { College of Staten } \\
\text { Island }\end{array}$ & & $\mathrm{BIO}$ & & $\begin{array}{c}\text { Medical } \\
\text { Technology, BIO, } \\
\text { Bioinformatics, } \\
\text { Ecology, Evolution, } \\
\text { Behavioral BIO, } \\
\text { Neuroscience, } \\
\text { Health Science }\end{array}$ & $\begin{array}{l}\text { BIO/Biotech, } \\
\text { Neuroscience and } \\
\text { Developmental } \\
\text { Disabilities }\end{array}$ & BIO, Biochemistry \\
\hline $\begin{array}{c}\text { Farmingdale State } \\
\text { University }\end{array}$ & & & MLT & MLS & & \\
\hline $\begin{array}{l}\text { Long Island } \\
\text { University }\end{array}$ & & & & $\begin{array}{l}\text { CLS, Biomedical } \\
\text { Science }\end{array}$ & $\begin{array}{c}\text { CLS, Biomedical } \\
\text { Science, Cardiovascular } \\
\text { Perfusion }\end{array}$ & \\
\hline SUNY at Buffalo & & & & $\begin{array}{c}\text { Medical } \\
\text { Technology, } \\
\text { Biotechnology }\end{array}$ & Biotechnology & \\
\hline $\begin{array}{c}\text { SUNY at } \\
\text { Stoneybrook }\end{array}$ & PBT & & & $\begin{array}{l}\text { CLS, MLT-CLS } \\
\text { online }\end{array}$ & Medical Molecular BIO & \\
\hline York College & & & & CLS, Health Science & PA & \\
\hline $\begin{array}{l}\text { University of North } \\
\text { Dakota }\end{array}$ & Histotech & & & $\begin{array}{l}\text { MLS, MLT-MLS } \\
\text { online }\end{array}$ & MLS & \\
\hline $\begin{array}{l}\text { Bowling Green } \\
\text { State University }\end{array}$ & $\begin{array}{l}\text { Food and } \\
\text { Nutrition }\end{array}$ & Public Health & & $\begin{array}{l}\text { MLS, Public Health, } \\
\text { Allied Health, } \\
\text { Applied Health } \\
\text { Science, Dietetics }\end{array}$ & $\begin{array}{l}\text { Food and Nutrition, } \\
\text { Health Services Admin }\end{array}$ & \\
\hline $\begin{array}{l}\text { Ohio Northern } \\
\text { University }\end{array}$ & & $\mathrm{BIO}$ & & $\begin{array}{c}\text { MLS, BIO, Mol } \\
\text { BIO, Forensic BIO, } \\
\text { Env and Field BIO }\end{array}$ & & \\
\hline $\begin{array}{l}\text { University of } \\
\text { Cincinnati }\end{array}$ & $\begin{array}{l}\text { Post-bac MLS, } \\
\text { Health } \\
\text { Information } \\
\text { Security }\end{array}$ & & & $\begin{array}{l}\text { MLS, MLT-MLS } \\
\text { online, Advanced } \\
\text { medical Imaging, } \\
\text { Respiratory therapy, } \\
\text { Health Information } \\
\text { Man }\end{array}$ & Health Informatics & \\
\hline
\end{tabular}




\begin{tabular}{|c|c|c|c|c|c|c|}
\hline University & Certificate & Minor & AA & BS & MS & Doctoral \\
\hline $\begin{array}{l}\text { University of } \\
\text { Toledo }\end{array}$ & & & & $\begin{array}{c}\text { Medical } \\
\text { Technology, BIO }\end{array}$ & $\mathrm{BIO}$ & $\mathrm{BIO}$ \\
\hline $\begin{array}{l}\text { Wright State } \\
\text { University }\end{array}$ & Post-bac MLS & BIO & & $\begin{array}{l}\text { CLS, BIO, Public } \\
\text { Health }\end{array}$ & $\mathrm{BIO}$ & \\
\hline $\begin{array}{l}\text { Youngstown State } \\
\text { University }\end{array}$ & & & $\begin{array}{l}\text { MLT, } \\
\text { EMT, } \\
\text { Medical } \\
\text { Assisting }\end{array}$ & $\begin{array}{l}\text { MLS, Allied Health, } \\
\text { Public Health, } \\
\text { Dental Hygiene, } \\
\text { Respiratory Care }\end{array}$ & $\begin{array}{l}\text { Health and Human } \\
\text { Services, Public Health, } \\
\text { Respiratory Care }\end{array}$ & \\
\hline $\begin{array}{l}\text { Northeastern State } \\
\text { University }\end{array}$ & & & & $\begin{array}{c}\text { MLS, MLT-MLS } \\
\text { online, Nursing, } \\
\text { Nutritional Science, } \\
\text { Speech Language } \\
\text { Pathology } \\
\end{array}$ & $\begin{array}{c}\text { Nursing, Speech } \\
\text { Language Pathology, PA, } \\
\text { OT }\end{array}$ & \\
\hline $\begin{array}{c}\text { Thomas Jefferson } \\
\text { University }\end{array}$ & & & & $\begin{array}{l}\text { MLS, } \\
\text { Biotechnology, } \\
\text { Cytotech }\end{array}$ & $\begin{array}{c}\text { Biotechnology, Cytotech, } \\
\text { MLS }\end{array}$ & \\
\hline $\begin{array}{l}\text { University of } \\
\text { Tennessee }\end{array}$ & $\begin{array}{l}\text { Histotech, } \\
\text { Clinical } \\
\text { Chemistry, } \\
\text { Micro } \\
\end{array}$ & & & $\begin{array}{l}\text { CLS, MLT-CLS } \\
\text { online }\end{array}$ & CLS, Cytotech & \\
\hline $\begin{array}{l}\text { Austin Peay State } \\
\text { University }\end{array}$ & PBT & & & $\begin{array}{l}\text { MLS, Radiologic } \\
\text { Tech }\end{array}$ & & \\
\hline $\begin{array}{l}\text { Tarleton State } \\
\text { University }\end{array}$ & & & $\begin{array}{l}\text { MLT, } \\
\text { Histotech }\end{array}$ & $\begin{array}{l}\text { MLS, Public Health, } \\
\text { Health Professions } \\
\text { Technology }\end{array}$ & MLS & \\
\hline $\begin{array}{c}\text { Texas A\&M } \\
\text { Corpus Christi }\end{array}$ & Post-bac MLS & $\mathrm{BIO}$ & & CLS, BIO & $\begin{array}{l}\text { BIO, Fisheries and } \\
\text { Mariculture }\end{array}$ & \\
\hline $\begin{array}{l}\text { Texas Southern } \\
\text { University }\end{array}$ & & & & $\begin{array}{c}\text { CLS, Health } \\
\text { Admin , HIM, } \\
\text { Respiratory therapy }\end{array}$ & Healthcare Admin & \\
\hline $\begin{array}{l}\text { Texas Tech } \\
\text { University }\end{array}$ & Post-bac MLS & & & CLS & Molecular Pathology, PA & \\
\hline UTMB & SBB & & & CLS & CLS & CLS \\
\hline UT-Rio Grande & & & & $\begin{array}{l}\text { CLS, Nursing, } \\
\text { Health Science } \\
\text { Technology }\end{array}$ & Health Science & \\
\hline University of. Utah & & & & MLS & & Medicine \\
\hline $\begin{array}{l}\text { Weber State } \\
\text { University }\end{array}$ & $\begin{array}{l}\text { Clinical Lab } \\
\text { Assisting }\end{array}$ & & MLS & $\begin{array}{l}\text { MLS, MLT-MLS } \\
\text { online }\end{array}$ & & \\
\hline $\begin{array}{l}\text { Virginia } \\
\text { Commonwealth } \\
\text { University }\end{array}$ & & & & CLS & CLS & $\begin{array}{c}\text { CLS Health } \\
\text { Related Science }\end{array}$ \\
\hline $\begin{array}{l}\text { University of } \\
\text { Vermont }\end{array}$ & & & & $\begin{array}{l}\text { MLS, Medical } \\
\text { Radiation, Health } \\
\text { Science }\end{array}$ & MLS & \\
\hline $\begin{array}{l}\text { University of } \\
\text { Wisconsin } \\
\text { Milwaukee }\end{array}$ & & & & $\begin{array}{l}\text { MLS, Biomedical } \\
\text { Science, Cytotech, } \\
\text { Public Health }\end{array}$ & Biomedical Science & \\
\hline $\begin{array}{l}\text { West Virginia } \\
\text { University }\end{array}$ & & & & MLS, Histotech & Pathologist Assistant & Medicine \\
\hline $\begin{array}{l}\text { West Liberty } \\
\text { University }\end{array}$ & & Gerontology & & $\begin{array}{c}\text { MLS, Nursing, } \\
\text { Psychology, Speech } \\
\text { Pathology, Dental } \\
\text { Hygiene }\end{array}$ & Psychology & \\
\hline $\begin{array}{l}\text { Inter-American } \\
\text { University of } \\
\text { Puerto Rico }\end{array}$ & Post-bac MT & & $\begin{array}{l}\text { Nursing, } \\
\text { Radiologic } \\
\text { Tech }\end{array}$ & $\begin{array}{l}\text { MT, Nursing, } \\
\text { Radiologic Tech }\end{array}$ & & \\
\hline $\begin{array}{l}\text { University of } \\
\text { Puerto Rico }\end{array}$ & & & $\begin{array}{c}\text { Dental } \\
\text { Asst, } \\
\text { Ophthalmic } \\
\text { Tech, } \\
\text { Radiologic } \\
\text { Tech }\end{array}$ & $\begin{array}{l}\text { MLS, Health Ed } \\
\text { Promotion, Health } \\
\text { Sciences, Vet Tech, } \\
\text { Nuclear Med Tech }\end{array}$ & & \\
\hline
\end{tabular}

PBT (Phlebotomy), MLS (Medical Laboratory Scientist), MT (Medical Technology), MLT (Medical Laboratory Technician), OT (Occupational Therapy), CLS (Clinical Laboratory Science), UT (University of Texas), UTMB (University of Texas Medical Branch), EMT (Emergency Medical Technician), PA (Physician Assistant), BIO (Biology), SBB (Specialist in Blood Banking), DGS (Diagnostic Genetic Science), HIM (Health Information Management), QEP (Quality Environment Proficiency), HMM (Hazardous Materials Management). 

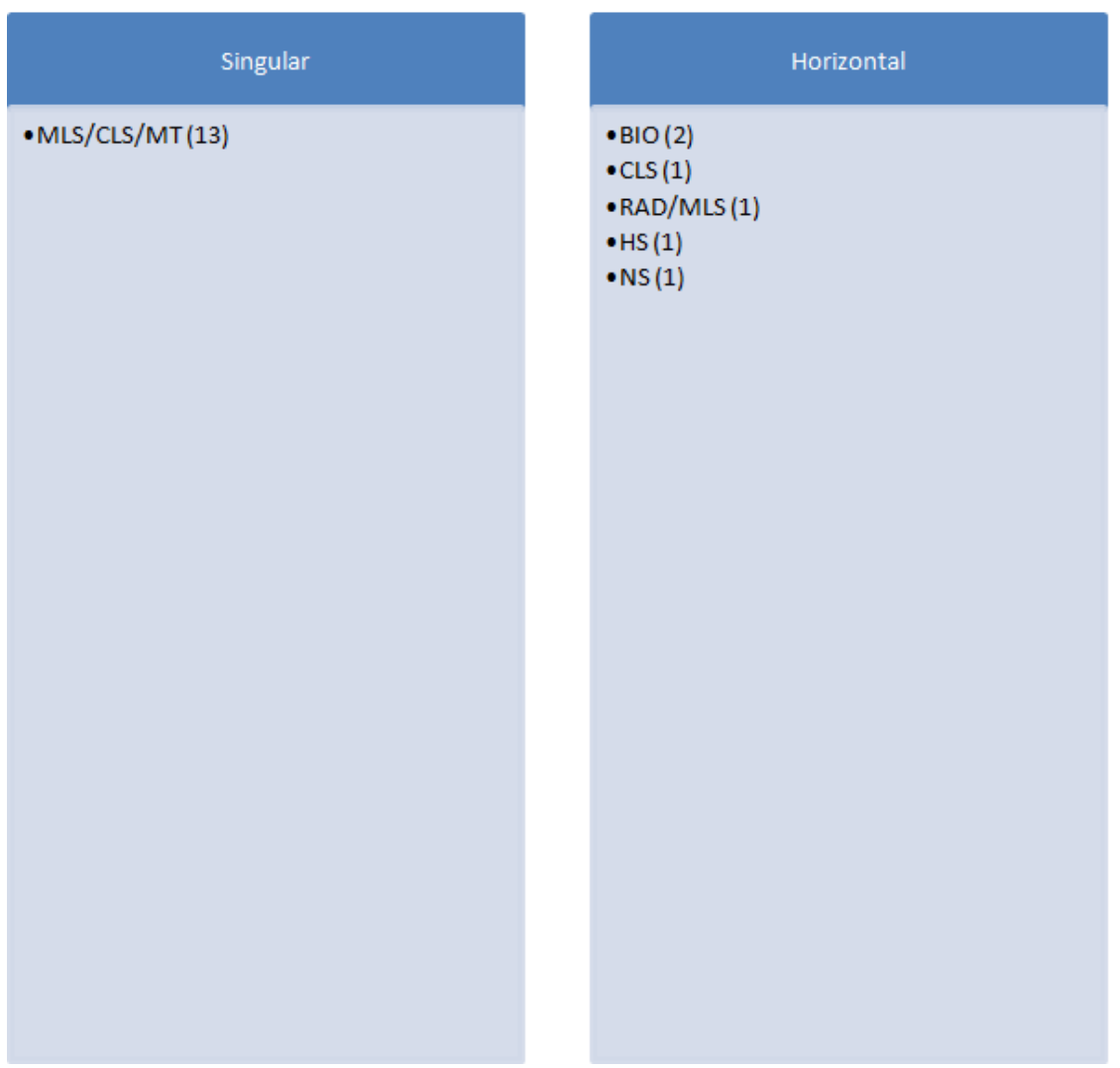

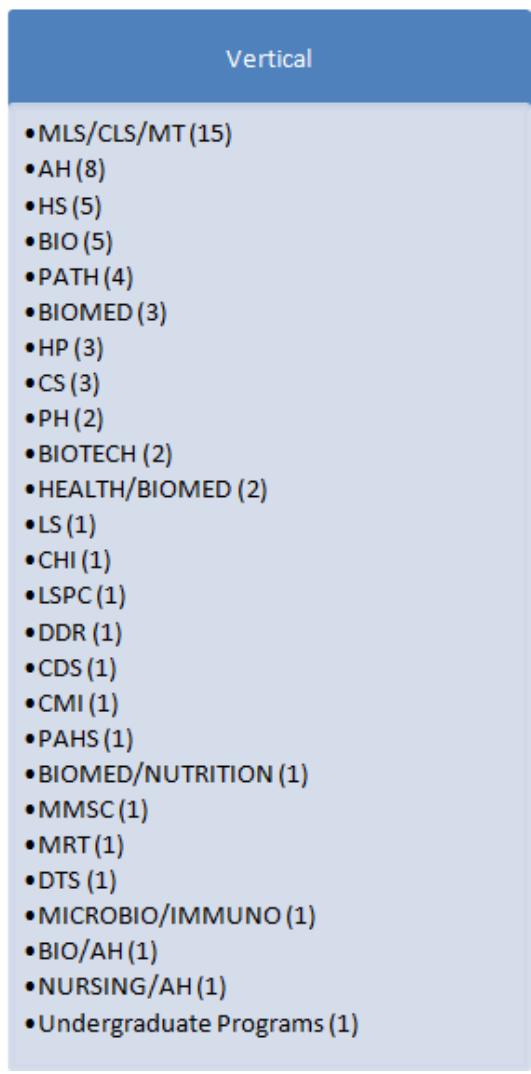

Figure 1. Academic Departments housing MLS Programs. MLS (Medical Laboratory Science), CLS (Clinical Laboratory Science), AH (Allied Health), BIO (Biology), RAD/MLS (Radiology and Medical Laboratory Science), HS (Health Sciences), NS (Natural Sciences), PATH (Pathology), BIOMED (Biomedical Sciences), HP (Health Professions), CS (Clinical Sciences), PH (Public Health), BIOTECH (Biotechnology), LS (Life Sciences), CHI (Clinical and Health Information), LSPC (Lab Sciences and Primary Care), DDR (Dental, Diagnostics, and Rehabilitation), CDS (Clinical and Diagnostic Sciences), CMI (Clinical and Medical Imaging), PAHS (Public/Allied Health Sciences), MMSC (Medical and Molecular Sciences), MRT (Medical and Research Technology), DTS (Diagnostic and Therapeutic Sciences)
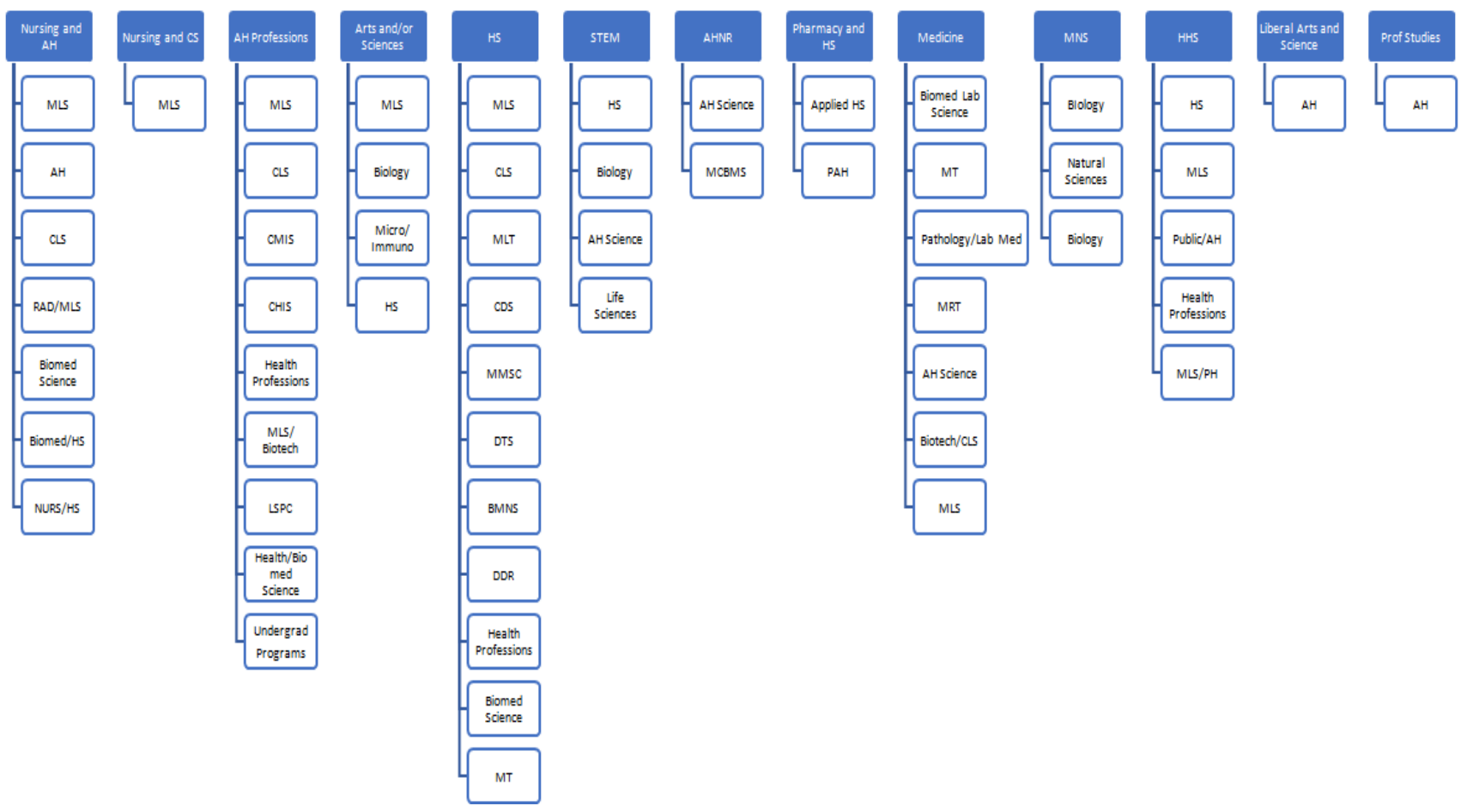

Figure 2. Colleges and Schools housing MLS programs. MT (Medical Technology), MRT (Medical and Research Technology), CLS (Clinical Laboratory Sciences), RAD (Radiological Science), HS (Health Sciences), CMIS (Clinical and Medical Imaging Sciences), CHIS (Clinical and Health Information Sciences), LSPC (Life Sciences and Primary Care), MLT (Medical Laboratory Technology), CDS (Clinical and Diagnostic Sciences), MMSC (Medical and Molecular Sciences), DTS (Diagnostic and Therapeutic Sciences), BMNS (Biomedical and Nutrition Sciences), DDR (Dental, Diagnostic, and Rehabilitation), AH (Allied Health), MCBMS (Molecular, Cellular, Biomedical Sciences), PAHS (Public and Allied Health Sciences), CS (Clinical Sciences), MNS (Math and Natural Sciences), HHS (Health and Human Services), PAH (Pharmacy Administration Health) 


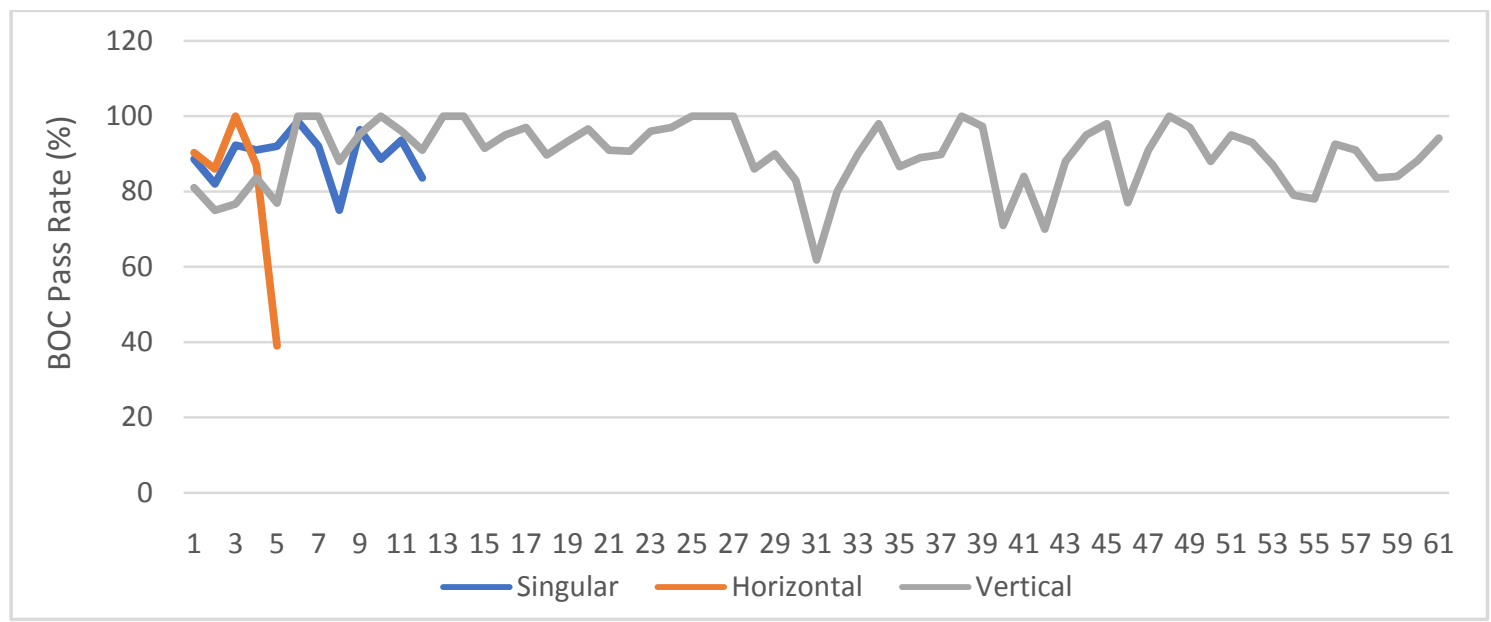

Figure 3. MLS ASCP BOC 3-Yr AVG Among Dept Models

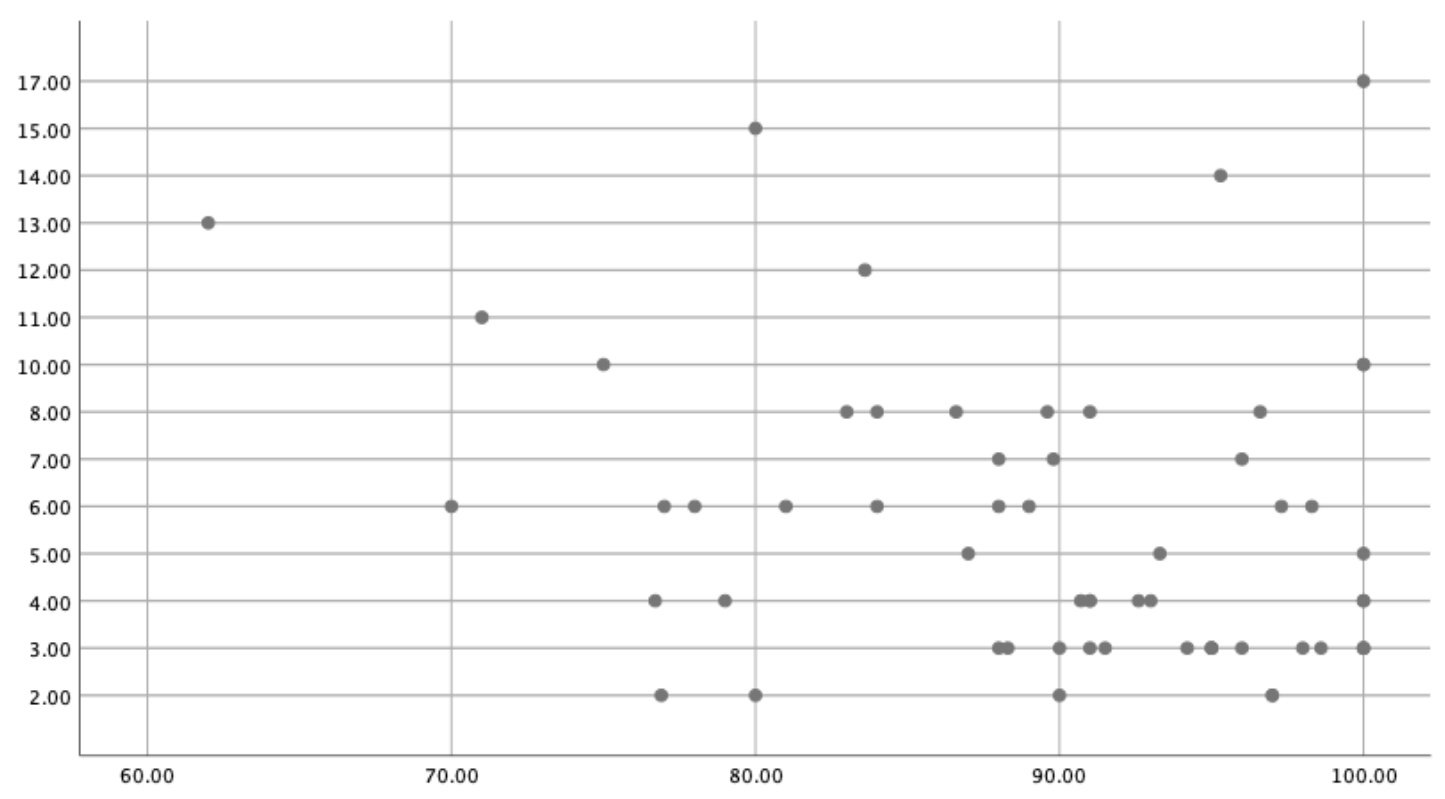

Avg BOC Pass Rate (\%)

Figure 4. Number of Programs vs Avg BOC Pass Rate (Vertical Model) $r=-.26$

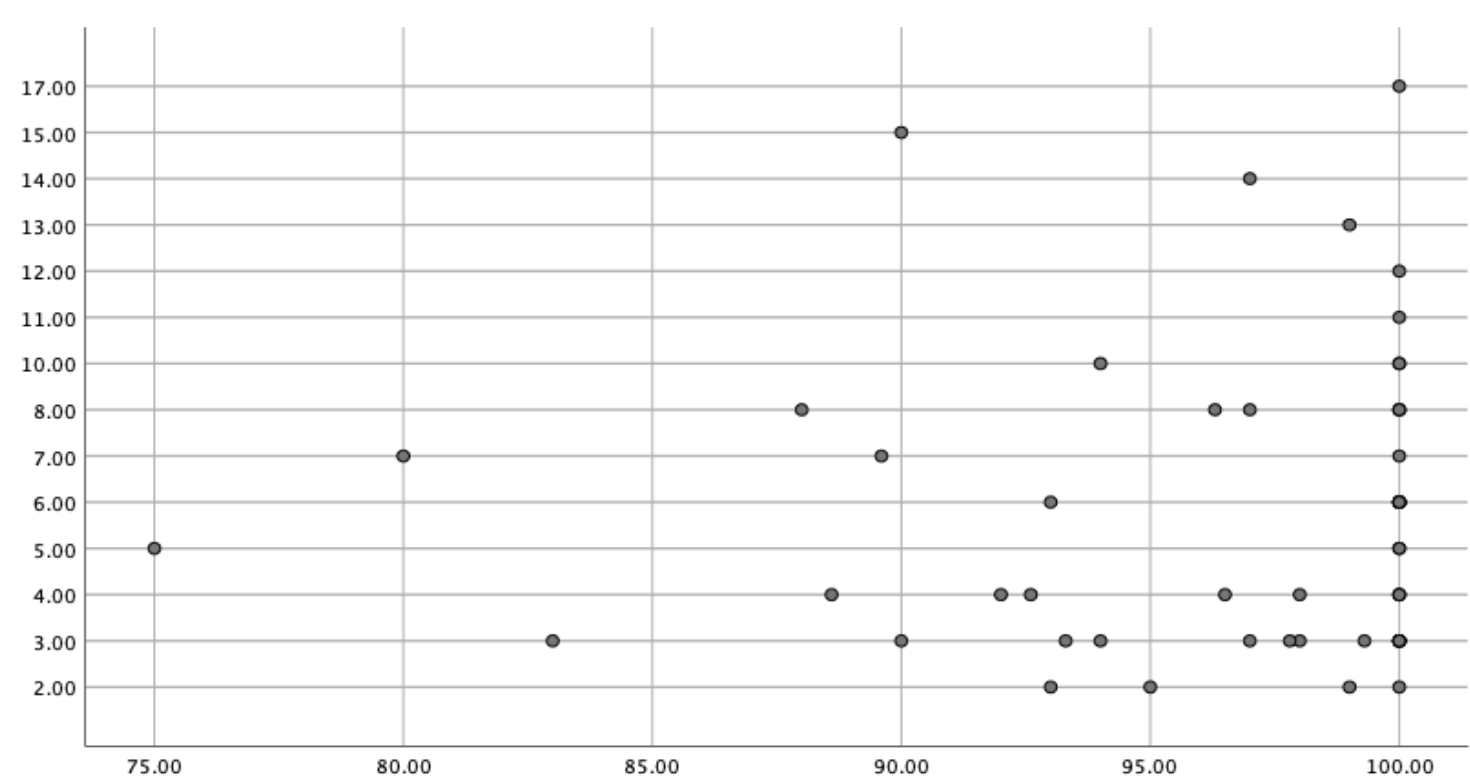

Avg Grad Rate (\%)

Figure 5. Number of Programs vs Avg Graduation Rate (Vertical Model) $r=.04$ 


\section{Discussion}

This study introduced three departmental models in mapping the current national landscape of universitybased MLS programs. The most common departmental model was Vertical with potential for interdisciplinary collaboration, shared resources, and increased enrollment for the department and college or school. The Horizontal model comprised the fewest MLS programs. Student outcomes were assessed respective of the three models. While there was no significant difference found with either the average 3-yr ASCP BOC pass rate nor the 3-yr average graduation rate among the three models, the most significant and interesting finding concerned the vertical model. A negative correlation coefficient and significant $\mathrm{P}$ value was found when assessing an association between the number of programs in a vertical oriented department and average 3-yr ASCP BOC pass rate; as the number of programs increase, the BOC pass rate decreases. Conversely, a positive correlation was found when assessing the number of programs in a department and average 3-yr graduation rate, however the $\mathrm{P}$ value was not significant.

The literature is devoid of comprehensive categorization of MLS programs offered in a university setting and effects on student outcomes in the sphere of models introduced. However, Wayne et al [4] posited a new paradigm was on the horizon concerning MLS programs where stand-alone programs, analogous to Singular programs in this study, were phasing out in favor of increased cooperation between disciplines in achieving outcomes influenced by ominous budget concerns [5], low student enrollment, dwindling clinical affiliations, and high cost of MLS programs. As evident in this study, Singular programs are in the minority in comparison with Vertical models. University administrators recognize the benefits of cooperation among disciplines in academic departments. While graduation rates were not a significant factor in the correlation coefficient analysis, the ASCP BOC pass rate appears to be a vulnerable outcome in the Vertical model. There are many factors, common to all department models, that may affect the BOC pass rate. These include screening of applicants, hiring qualified faculty, and adequate resources, to name a few. A factor that is unique to Vertical model is serving other programs in the department as well as the MLS program. Increased administrative tasks, advising, teaching classes in multiple disciplines may lead to reallocation of time needed to plan and implement strategies geared toward increasing BOC pass rates in a vertical-structured department.

An example of an MLS program in a Vertical department that has been successful in maintaining favorable BOC pass rates is the Department of Medical and Molecular Sciences (MMSC) at the University of Delaware (UD). The MLS program is structured in a $2+2$ format. As depicted in Table 3, the MMSC department offers certificate programs, minor degrees, multiple baccalaureate and Master's programs, and a PhD program. The MT program began in 1948 in the Biology department, College of Arts \& Sciences.
Table 3. University of Delaware's MLS Program History

\begin{tabular}{|c|l|}
\hline 1948 & $\begin{array}{l}\text { MT Program began in the Biology Department, College of } \\
\text { Arts and Sciences }\end{array}$ \\
\hline 1995 & $\begin{array}{l}\text { MT Department established in College of Health and Nursing } \\
\text { Science }\end{array}$ \\
\hline 2005 & $\begin{array}{l}\text { College of Health and Nursing science became the College of } \\
\text { Health Sciences }\end{array}$ \\
\hline 2008 & Language of Medicine offered as open enrollment course \\
\hline 2009 & Forensic Science offered as open enrollment course \\
\hline 2010 & Medical Diagnostics Minor \\
\hline 2012 & $\begin{array}{l}\text { Changed Department name to Medical Laboratory } \\
\text { Sciences/PhD in Medical Sciences offered }\end{array}$ \\
\hline 2013 & BS in Medical Diagnostics (Pre PA option) \\
\hline 2015 & Forensic Science and Molecular Diagnostics Minors \\
\hline 2016 & MS in Medical Sciences and BS in AMBB \\
\hline 2017 & Certificate in Molecular Diagnostics \\
\hline 2018 & $\begin{array}{l}\text { Changed Department name to Medical and Molecular } \\
\text { Sciences; Minor in Genetic Counseling offered }\end{array}$ \\
\hline 2019 & \begin{tabular}{l}
$4+1$ MS in AMBB and MLS \\
\hline
\end{tabular} \\
\hline
\end{tabular}

On its path to the MMSC department it transitioned from a Vertical department to a singular department and back to a Vertical department (Table 3). The impetus for addition and diversification of programs was implementation of Responsibility Based Budgeting (RBB) in 2010 which rendered colleges and departments accountable for their revenues and expenditures. As a result, the MT department's focus turned to adding programs that complemented its curriculum and resources. As depicted in Table 3, incremental changes and evaluation thereof gave way to the addition and diversification of degree and certificate programs. Open enrollment courses precipitated offering minors in Medical Diagnostics, Forensic science, and Molecular Diagnostics. This led to development of baccalaureate programs in Medical Diagnostics (MDD) and Applied Molecular Biology and Biotechnology (AMBB). Success of minors and new majors led to creation of Master of Science degrees in Medical Science, AMBB, and MLS. The minor degree in Genetic Counseling encompasses aspects of all programs offered in the department. The changes and strategies implemented to increase enrollment were successful; enrollment for MLS majors increased by $34 \%$ from 2009 to 2018. In addition, the department was now interdisciplinary on a variety of levels. The common lecture courses taken by the MDD (major and minor), MDD Pre-PA, and MLS students render these classes gateways to interprofessional collaboration as students transition from their chosen academic programs to professional practice. Another benefit to undergraduate students is having discussions with graduate students on their interdisciplinary experiences and research interests, which may influence their decision toward pursuit of an advanced degree. Laboratories in the department are shared among the various disciplines inside and outside of the department. As many as four disciplines may utilize one of three student laboratories in the Fall and Spring semesters. There are ten full-time faculty, two laboratory coordinators, one educational clinical coordinator, and three administrative staff to support all programs. Three faculty serve as Directors of the MLS, MDD, and MDD Pre-PA programs. The Department Chair serves as the 
Director of the BS in AMBB program and graduate programs. Faculty teach courses in their area of expertise and most teach in both the undergraduate and graduate programs. An Introduction to MLS course is taught to high school students in a face to face format giving faculty the opportunity to educate younger generations on the MLS profession as well as a conclave for recruitment and potential pipeline for the MLS program. Successful strategies in place to help maintain BOC pass rates above 90 percent include comprehensive (students must earn a $65 \%$ or better) and end of rotation exams after each clinical rotation, utilization of MediaLab LabCE where students answer approximately 700 questions in bacteriology, mycology, parasitology, immunology, blood bank, hematology, body fluids, urinalysis, clinical chemistry, and laboratory operations. In their last semester, students are also assigned a case study with relevance to one MLS discipline to present to their peers and faculty, in addition to writing a paper on the case. Future strategies may include setting a minimum grade for the end of rotation exam and for LabCE assignments.

The second most common department model is Singular. These MLS programs conceivably allocate all resources and time toward recruitment, maintaining NAACLS accreditation, and positive student outcomes for a single program. An example of an MLS program in a singular model is the Department of Clinical and Medical Sciences at Auburn University Montgomery (AUM) in Montgomery, Alabama. This program began in 1977 and interestingly transitioned from a horizontal model to a singular model, moving from the department of Biology in the School of Sciences to its current department in the College of Nursing and Health Sciences. The MLS program is structured in a $2+2$ format (online and on campus). Strategies employed toward increasing BOC pass rates include students taking an exam after each clinical rotation of clinical chemistry, hematology, immunohematology, and microbiology. Once students complete their clinical rotations they take a series of 100 question multiple choice exams in an online format and must attain a score of $70 \%$ to pass the comprehensive review course. After each of these exams on campus students have quality time to discuss questions with faculty and their peers in a quiet environment that is cohesive and staunchly focused on students passing the BOC exam. An advantage to AUMs comprehensive review course is the students begin the course in the summer after successful completion of all clinical rotations which allows them to solely focus on review questions that cover all disciplines for MLS in close proximity to taking the BOC exam.

The horizontal model was least utilized model in the realm of MLS programs. Departments are characterized as interdisciplinary offering at least one other BS program aside from MLS which may not be an allied health program, e.g Biology. An example of an MLS program exhibiting the horizontal model is the Department of Radiologic and MLS in the College of Nursing and Health Professions at McNeese State University (Lake Charles, LA). The structure of the MLS program is $3+1$ where students spend their first 3 years on campus and last year in a clinical internship. The MLS program began in 1954 and was situated in the biology department which could be classified as horizontal. In 2015, it transitioned to its current department. The reason for the change was to place the MLS program in an environment that was more related to allied health where programs could share resources and learning could be interdisciplinary. As the transition to its new department is fairly recent, ideas for sharing resources are still in the planning stages. The Program Director for Radiological Sciences serves as the Department Head which conserves cost associated with hiring a Chair for the department. The department has two program directors, five faculty and one administrative assistant. Strategies employed to maintain BOC pass rates above 90 percent include MediaLab LabCE quizzes and end of rotation exams in the clinical internship. McNeese State University provides an example of an MLS program has remained horizontal despite switching from a biology department to the Radiologic and MLS department.

Limitations of this study include ASCP BOC pass rates and Graduation rates not accessible from the MLS Programs' website. Of the 66 Vertical departments, 5 BOC pass rates and 7 graduation rates were not available from the programs' website. Of the 14 Singular departments, 2 BOC pass rates and graduation rates were not available from the programs' website. Of the 6 Horizontal departments, 1 MLS program displayed their 5 -yr BOC pass rate instead of 3-yr pass rate and was not included in the analysis.

The national landscape of university departments housing MLS programs is broadly heterogenous with regard to the three models introduced, the variety of department names, and other programs offered in the horizontal and vertical models. The academic department remains the bastion of degree program administration in universities despite criticism and calls for its interment. In 1971, Dael Wolfle who served as Director for the Advancement of Science, posited the academic department should be retired in favor of academic divisions to more efficiently dissect and solve problems in academia and facilitate interdisciplinary collaboration among faculty [6]. In 2018, Irani argued that the department model should be replaced not by academic divisions but by interdisciplinary centers that perpetuate more cooperation and less confinement; where teaching and research is strengthened, course development is more fluid, and interdisciplinary collaboration is the norm [7]. Despite these views, the academic landscape has not been redrawn toward depletion of departments. Interdisciplinary collaboration is largely individualist and not contingent on any one type of organizational structure. Faculty and researchers from across colleges attend seminars, serve on college and university committees, collaborate on research projects, and mentor students outside of their department. These interactions have influenced administrators toward widespread adoption of the vertical model where students are offered a plethora of programs to pursue, resources are consolidated, faculty roles are expanded, and interdisciplinary collaboration has become a department's silver lining of productivity, growth, and ultimately survival.

\section{Conclusion}

This study introduced three novel models of academic departments housing MLS programs tethered to a 
landscape that has become overwhelmingly interdisciplinary across the United States. The majority of departments were categorized as Vertical, followed by Singular and Horizontal. Analysis of variance statistics revealed there was not a statistical significant difference among the three department models with regard to average BOC pass rates and graduation rates. The most compelling finding of this study was a negative correlation in the vertical model with regard to quantity of programs offered and average BOC pass rates. The example outlined for the MMSC department at the University of Delaware may be reminiscent of other vertical departments in striking the right balance in number of programs to offer, hiring and retaining qualified faculty and staff, employing strategies for recruitment and retention while maintaining positive student outcomes. Vertical departments are likely to increase across college campuses in the next ten years. Today's models serve as a guide for what combination of programs is deemed the most efficient and enduring for colleges while providing quality education and enriching opportunities to our students.

\section{References}

[1] Clough GC, Stein BR, Neurath H, Zierler KL. University Organization. Science. 1971; 174(4016): 1279-1280.

[2] Storberg-Walker J, Torraco R. Change in Higher Education: a multidiscipline approach. Academy of Human Resource Development International Conference 2004; 3(7): 811-818.

[3] Bennett A, Garcia E, Schulze $M$ et al. Building a Laboratory Workforce to Meet the Future: ASCP Task Force on the Laboratory Professionals Workforce. AJCP 2014; 141: 154-167.

[4] Bruce W, Bruce JM, Paur RA. A New Paradigm for Clinical Laboratory Science Education: A Case Study for Survival and Growth. Clinical Laboratory Science 1997; 10(1): 38-42.

[5] Rudmann SV. University-Based Clinical Laboratory Science Programs: Strategies for Survival. Clinical Laboratory Science 1995; 8(2): 90-94.

[6] Wolfle D. The Supernatural Department. Science 1971; 173(3992): 109.

[7] Irani Z. The University of the future will be Interdisciplinary. The Guardian. 2018 Jan 24. 